Proyecciones Journal of Mathematics

Vol. 35, No 4, pp. 405-415, December 2016.

Universidad Católica del Norte

Antofagasta - Chile

\title{
Some results on skolem odd difference mean labeling
}

\author{
P. Jeyanthi \\ Govindammal Aditanar College for Women, India \\ R. Kalaiyarasi \\ Dr. Sivanthi Aditanar College of Engineering, India \\ D. Ramya \\ Government Arts College for Women, India \\ and \\ T. Saratha Devi \\ G. Venkataswamy Naidu College, India \\ Received : November 2015. Accepted : October 2016
}

\begin{abstract}
Let $G=(V, E)$ be a graph with $p$ vertices and $q$ edges. A graph $G$ is said to be skolem odd difference mean if there exists a function $f: V(G) \rightarrow\{0,1,2,3, \ldots, p+3 q-3\}$ satisfying $f$ is $1-1$ and the induced map $f^{*}: E(G) \rightarrow\{1,3,5, \ldots, 2 q-1\}$ defined by $f^{*}(e)=\left\lceil\frac{|f(u)-f(v)|}{2}\right\rceil$ is a bijection. A graph that admits skolem odd difference mean labeling is called skolem odd difference mean graph. We call a skolem odd difference mean labeling as skolem even vertex odd difference mean labeling if all vertex labels are even. A graph that admits skolem even vertex odd difference mean labeling is called skolem even vertex odd difference mean graph.

In this paper we prove that graphs $B(m, n): P_{w},\left\langle P_{m} \tilde{o} S_{n}\right\rangle, m P_{n}$, $m P_{n} \cup t P_{s}$ and $m K_{1, n} \cup t K_{1, s}$ admit skolem odd difference mean labeling. If $G(p, q)$ is a skolem odd differences mean graph then $p \geq q$. Also, we prove that wheel, umbrella, $B_{n}$ and $L_{n}$ are not skolem odd difference mean graph.
\end{abstract}

Keywords : Skolem difference mean labeling, skolem odd difference mean labeling, skolem odd difference mean graph, skolem even vertex odd difference mean labeling, skolem even vertex odd difference mean graph.

AMS Subject Classification: $05 C 78$. 


\section{Introduction}

Throughout this paper by a graph we mean a finite, simple and undirected one. The vertex set and the edge set of a graph $G$ are denoted $V(G)$ and $E(G)$ respectively. Terms and notations not defined here are used in the sense of Harary [1]. A graph labeling is an assignment of integers to the vertices or edges or both, subject to certain conditions. There are several types of labeling. An excellent survey of graph labeling is available in [2]. The concept of mean labeling was introduced by Somasundaram and Ponraj [8]. A graph $G(V, E)$ with $p$ vertices and $q$ edges is called a mean graph if there is an injective function $f$ that maps $V(G)$ to $\{0,1,2, \ldots, q\}$ such that for each $u v$, labeled with $\frac{f(u)+f(v)}{2}$ if $f(u)+f(v)$ is even and $\frac{f(u)+f(v)+1}{2}$ if $f(u)+f(v)$ is odd. Then the resulting edge labels are distinct. The notion of odd mean labeling was due to Manickam and Marudai [3]. Let $G(V, E)$ be a graph with $p$ vertices and $q$ edges. A graph $G$ is said to be odd mean graph if there exists a function $f: V(G) \rightarrow\{0,1,2,3, \ldots, 2 q-1\}$ satisfying $f$ is $1-1$ and the induced map $f^{*}: E(G) \rightarrow\{1,3,5, \ldots, 2 q-1\}$ defined by

$$
f^{*}(u v)=\left\{\begin{array}{c}
\frac{f(u)+f(v)}{2} \text { if } f(u)+f(v) \text { is even } \\
\frac{f(u)+f(v)+1}{2} \text { if } f(u)+f(v) \text { is odd }
\end{array}\right. \text { is a bijection. The }
$$

function $f$ is called an odd mean labeling. In [3], they studied the mean labeling of some standard graphs.

Murugan and Subramaniam [4] introduced the concept of skolem difference mean labeling and some standard results on skolem difference mean labeling were proved in [5] and [6]. A graph $G(V, E)$ with $p$ vertices and $q$ edges is said to have skolem diffference mean labeling if it is possible to label the vertices $x \in V$ with distinct elements $f(x)$ from $1,2,3, \ldots, p+q$ in such a way that for each edge $e=u v$, let $f^{*}(e)=\left\lceil\frac{|f(u)-f(v)|}{2}\right\rceil$ and the resulting labels of the edges are distinct and are from $1,2,3, \ldots, q$. A graph that admits skolem difference mean labeling is called a skolem difference mean graph. Motivated by the concept of skolem difference mean labeling Ramya et al. [7] introduced the concept of skolem odd difference mean labeling. A graph $G$ is said to have skolem odd difference mean labeling if there exists a function $f: V(G) \rightarrow\{0,1,2,3, \ldots, p+3 q-3\}$ satisfying that $f$ is $1-1$ and the induced map $f^{*}: E(G) \rightarrow\{1,3,5, \ldots, 2 q-1\}$ defined by $f^{*}(e)=\left\lceil\frac{|f(u)-f(v)|}{2}\right\rceil$ is a bijection. A graph that admits skolem odd difference mean labeling is called skolem odd difference mean graph. This is an extension work on skolem odd difference mean labeling. We use the 
following definitions in the subsequent section.

Definition 1.1. Let $P_{w}: v_{1}, v_{2}, \ldots, v_{w}$ be a path. The graph $B(m, n): P_{w}$ is obtained by identifying the central vertices of the star graphs $K_{1, m}$ and $K_{1, n}$ with $v_{1}$ and $v_{w}$ respectively.

Definition 1.2. Let $P_{m}$ be a path on $m$ vertices. The graph $\left\langle P_{m} \tilde{o} S_{n}\right\rangle$ is obtained from $P_{m}$ and $m$ copies of $K_{1, n}$ by joining the central vertex of $i^{\text {th }}$ copy of $K_{1, n}$ with $i^{\text {th }}$ vertex of $P_{m}$ by an edge.

Definition 1.3. The graph $m P_{n}$ is the disjoint union of $m$ copies of the path $P_{n}$.

Definition 1.4. The wheel graph $W_{n}$ is a graph with $n$ vertices $(n \geq 4)$, formed by connecting a single vertex to all vertices of a $(n-1)$ cycle.

Definition 1.5. A graph is obtained from a fan by joining a path of length $m, P_{m}$ to a middle vertex of a path $P_{n}$ in fan $F_{n}$ is called an umbrella graph and denoted by $U(m, n)$.

Definition 1.6. The book graph $B_{m}$ is defined as the cartesian product graph $S_{m+1} \times P_{2}$, where $S_{m}$ is a star graph and $P_{2}$ is the path graph on two vetices.

Definition 1.7. The cartesian product of two paths is known as grid graph $P_{m} \times P_{n}$. In particular the graph $L_{n}=P_{m} \times P_{2}$ is known as ladder graph.

\section{Skolem Odd Difference Mean Graphs}

In this section we prove that graphs $B(m, n): P_{w},\left\langle P_{m} \tilde{o} S_{n}\right\rangle, m P_{n}, m P_{n} \cup t P_{s}$ and $m K_{1, n} \cup t K_{1, s}$ admit skolem odd difference mean labeling. If $G(p, q)$ is a skolem odd differences mean graph then $p \geq q$. Also, we prove that wheel, umbrella, $B_{n}$ and $L_{n}$ are not skolem odd difference mean graph.

Theorem 2.1. The graph $B(m, n): P_{w}$ is a skolem odd difference mean graph. 
Proof. Let $V\left(B(m, n): P_{w}\right)=\left\{u_{i}, v_{j}, t_{k}: 1 \leq i \leq m, 1 \leq j \leq n, 1 \leq\right.$ $k \leq w\}$ and $E\left(B(m, n): P_{w}\right)=\left\{t_{1} u_{i}, t_{w} v_{j}, t_{k} t_{k+1}: 1 \leq i \leq m, 1 \leq j \leq\right.$ $n, 1 \leq k \leq w-1\}$ be the set of vertices and edges of the graph $B(m, n): P_{w}$. Define $f: V\left(B(m, n): P_{w}\right) \rightarrow\{0,1,2,3, \ldots, p+3 q-3=4(m+n+w)-6\}$ as follows:
$f\left(t_{2 i-1}\right)=4(i-1)$
$f\left(t_{2 i}\right)=4(n+w-i)-2$
for $1 \leq i \leq\left\lceil\frac{w}{2}\right\rceil$,
$f\left(u_{i}\right)=4(m+n+w-i)-2$
for $1 \leq i \leq\left\lfloor\frac{w}{2}\right\rfloor$,
$f\left(v_{i}\right)=2 w+4(i-1)$
for $1 \leq i \leq m$,
for $1 \leq i \leq n$.

Let $e_{i}=t_{i} t_{i+1} \quad$ for $1 \leq i \leq w-1$.

For each vertex label, the induced edge label $f^{*}$ is defined as follows:

$f^{*}\left(e_{i}\right)=2(n+w-i)-1 \quad$ for $1 \leq i \leq w-1$,

$f^{*}\left(t_{1} u_{i}\right)=2(m+n+w-i)-1 \quad$ for $1 \leq i \leq m$,

$f^{*}\left(t_{w} v_{i}\right)=2(n-i)+1 \quad$ for $1 \leq i \leq n$.

It can be verified that $f$ is a skolem odd difference mean labeling of $B(m, n): P_{w}$. The skolem odd difference mean labeling of $B(3,4): P_{5}$ is shown in Figure 1.

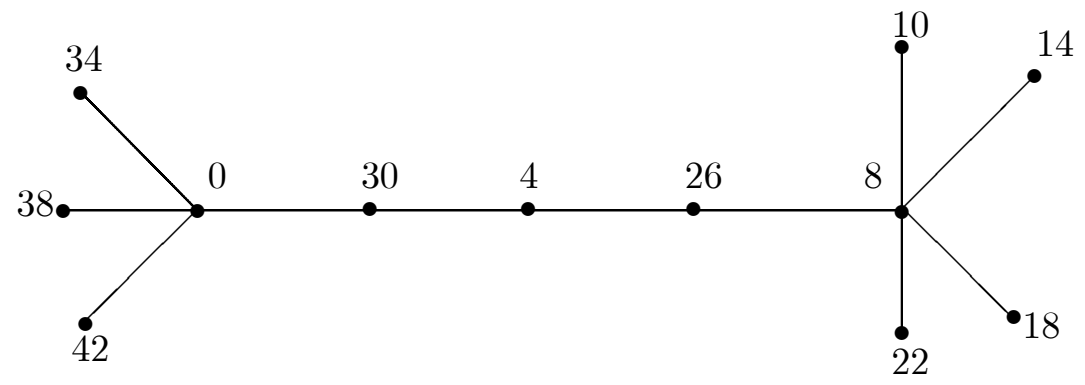

Figure 1 : Skolem odd difference mean labeling of $B(3,4): P_{5}$

Theorem 2.2. The graph $\left\langle P_{m} \tilde{o} S_{n}\right\rangle$ is a skolem odd difference mean graph.

Proof. Let $w_{j}, u_{j}, v_{i}^{j}(1 \leq j \leq m, 1 \leq i \leq n)$ be the vertices of $\left\langle P_{m} \tilde{o} S_{n}\right\rangle$. $w_{j} u_{j}, u_{k} u_{k+1}, w_{j} v_{i}^{j}: 1 \leq j \leq m, 1 \leq k \leq m-1,1 \leq i \leq n$ be the edges of $\left\langle P_{m} \tilde{o} S_{n}\right\rangle$. 
Define $f: V\left(\left\langle P_{m} \tilde{o} S_{n}\right\rangle\right) \rightarrow\{0,1,2,3, \ldots, p+3 q-3=4 m n+8 m-6\}$ as follows:

$f\left(u_{2 i-1}\right)=4 n+4(n+2)(i-1)$

$f\left(u_{2 i}\right)=4(n+2)(m-i+1)-10$

$f\left(w_{2 j-1}\right)=4(n+2)(m-j+1)-6$

$f\left(w_{2 j}\right)=4(n+1)+4(n+2)(j-1)$

for $1 \leq i \leq\left\lceil\frac{m}{2}\right\rceil$,

for $1 \leq i \leq\left\lfloor\frac{m}{2}\right\rfloor$,

for $1 \leq j \leq\left\lceil\frac{m}{2}\right\rceil$

$f\left(v_{i}^{j}\right)=2(n+2)(j-1)+4(i-1)$

for $1 \leq j \leq\left\lfloor\frac{m}{2}\right\rfloor$,

and $j$ is odd,

$f\left(v_{i}^{j}\right)=2(n+2)(2 m+2-j)-2(2 i+5) \quad$ for $1 \leq i \leq n, 1 \leq j \leq m$ and $j$ is even.

Let $e_{i}^{j}=w_{j} v_{i}^{j}$ for $1 \leq i \leq n, 1 \leq j \leq m$ and $e_{j}=w_{j} u_{j}$ for $1 \leq j \leq m$.

For each vertex labeling, the induced edge label $f^{*}$ is defined as follows. $f^{*}\left(u_{j} u_{j+1}\right)=2(n+2)(m-j+1)-2 n-5$ for $1 \leq j \leq m-1$, $f^{*}\left(e_{j}\right)=2(n+2)(m-j+1)-2 n-3 \quad$ for $1 \leq j \leq m$ and $j$ is odd, $f^{*}\left(e_{j}\right)=2(n+2)(m-j+2)-2 n-7 \quad$ for $1 \leq j \leq m$ and $j$ is even, $f^{*}\left(e_{i}^{j}\right)=2(n+2)(m-j+1)-2 i-1 \quad$ for $1 \leq i \leq n, 1 \leq j \leq m$ and $j$ is odd,

$f^{*}\left(e_{i}^{j}\right)=2(n+2)(m-j+2)-2(n+i)-7 \quad$ for $1 \leq i \leq n, 1 \leq j \leq m$ and $j$ is even.

It can be verified that $f$ is a skolem odd difference mean labeling of $\left\langle P_{m} \tilde{o} S_{n}\right\rangle$. The skolem odd difference mean labeling of $\left\langle P_{4} \tilde{o} S_{3}\right\rangle$ is shown in Figure 2.

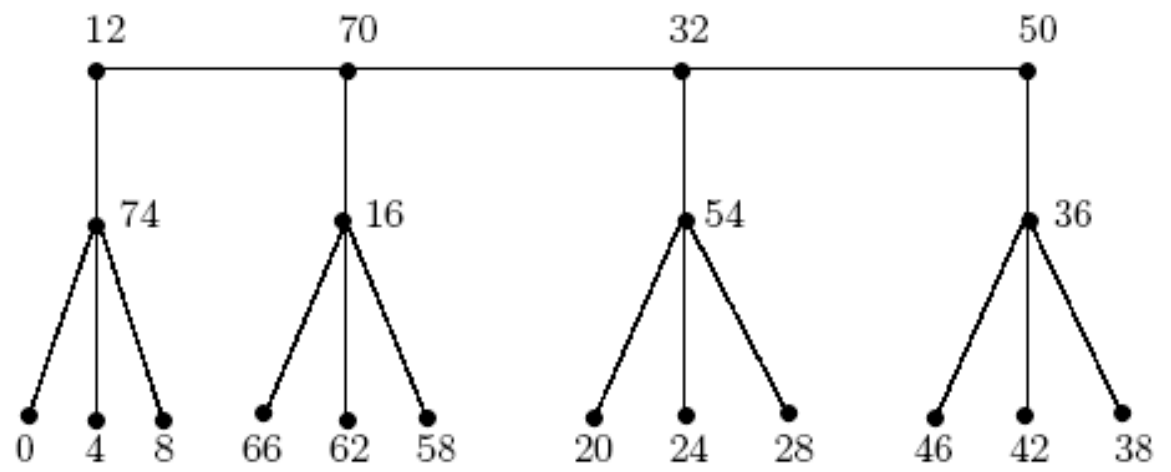

Figure 2: Skolem odd difference mean labeling of $\left\langle P_{4} \tilde{o} S_{3}\right\rangle$ 
Theorem 2.3. The graph $m P_{n}$ is a skolem odd difference mean graph.

Proof. Let $v_{1}^{1}, v_{2}^{1}, \ldots, v_{n}^{1}$ and $v_{1}^{2}, v_{2}^{2}, \ldots, v_{n}^{2}$ upto $v_{1}^{m}, v_{2}^{m}, \ldots, v_{n}^{m}$ be the vertices of the graph $m P_{n}$.

Let $V\left(m P_{n}\right)=\left\{v_{i}^{j}: 1 \leq i \leq n, 1 \leq j \leq m\right\}$ and $E\left(m P_{n}\right)=\left\{v_{i}^{j} v_{i+1}^{j}: 1 \leq\right.$ $i \leq n-1,1 \leq j \leq m\}$.

Define $f: V\left(m P_{n}\right) \rightarrow\{0,1,2, \ldots, 4 m n-3(m+1)\}$ as follows:

When $n$ is odd and $m$ is either odd or even,

$f\left(v_{2 i-1}^{j}\right)=(m-1)+2 n(j-1)+4(i-1) \quad$ for $1 \leq i \leq\left\lceil\frac{n}{2}\right\rceil$,

$1 \leq j \leq m$,

$f\left(v_{2 i}^{j}\right)=4 m n-3(m+1)-2(n-2)(j-1)-4(i-1)$ for $1 \leq i \leq\left\lfloor\frac{n}{2}\right\rfloor, 1 \leq j \leq m$.

When $n$ is even and $m$ is either odd or even,

$f\left(v_{2 i-1}^{j}\right)=(m-1)+2(n-1)(j-1)+4(i-1) \quad$ for $1 \leq i \leq\left\lceil\frac{n}{2}\right\rceil$,

$1 \leq j \leq m$,

$f\left(v_{2 i}^{j}\right)=4 m n-3(m+1)-2(n-1)(j-1)-4(i-1)$ for $1 \leq i \leq\left\lfloor\frac{n}{2}\right\rfloor, 1 \leq j \leq m$.

For each vertex labeling, the induced edge label $f^{*}$ is defined as follows: $f^{*}\left(v_{i}^{j} v_{i+1}^{j}\right)=2(n-1)(m-j+1)-2 i+1 \quad$ for $1 \leq i \leq n-1$, $1 \leq j \leq m$.

It can be verified that $f$ is a skolem odd difference mean labeling of $m P_{n}$.

The skolem odd difference mean labeling of $3 P_{4}$ is shown in Figure 3.

Theorem 2.4. The graph $m P_{n} \cup t P_{s}$ is a skolem odd difference mean graph if $m, n, t, s \geq 1$.

Proof. Let $v_{1}^{1}, v_{2}^{1}, v_{3}^{1}, \ldots, v_{n}^{1}$ and $v_{1}^{2}, v_{2}^{2}, v_{3}^{2}, \ldots, v_{n}^{2}$ upto $v_{1}^{m}, v_{2}^{m}, v_{3}^{m}, \ldots, v_{n}^{m}$ and $u_{1}^{1}, u_{2}^{1}, u_{3}^{1}, \ldots, u_{s}^{1}$ and $u_{1}^{2}, u_{2}^{2}, u_{3}^{2}, \ldots, u_{s}^{2}$, upto $u_{1}^{t}, u_{2}^{t}, u_{3}^{t}, \ldots, u_{s}^{t}$ be the vertices of the graph $m P_{n} \cup t P_{s}$. Let $E\left(m P_{n} \cup t P_{s}\right)=\left\{v_{i}^{j} v_{i+1}^{j}: 1 \leq i \leq\right.$ $n-1,1 \leq j \leq m$ and $\left.u_{i}^{j} u_{i+1}^{j}: 1 \leq i \leq s-1,1 \leq j \leq t\right\}$. 


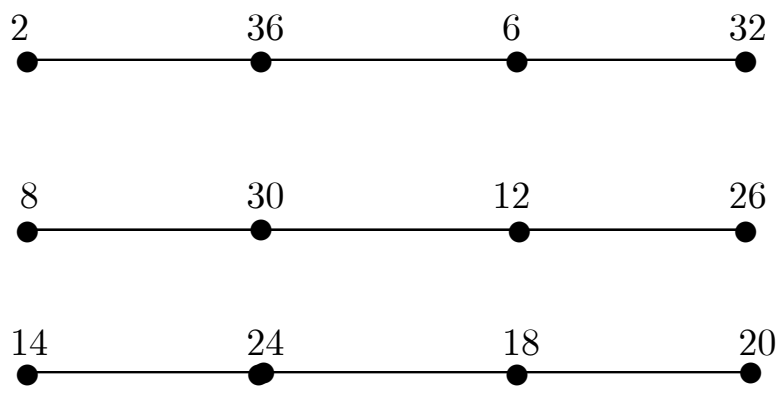

Figure 3 : Skolem odd difference mean labeling of $3 P_{4}$

Define $f: V\left(m P_{n} \cup t P_{s}\right) \rightarrow\{0,1,2, \ldots, 4(m n+t s)-3(m+t+1)\}$ as follows:

$f\left(v_{2 i-1}^{j}\right)=2+2(n-1)(j-1)+4(i-1) \quad$ for $1 \leq j \leq m$,

$1 \leq i \leq \frac{n}{2}$ and $n$ is even,

$f\left(v_{2 i}^{j}\right)=4 m n-4 m-2(n-1)(j-1)-4(i-1) \quad$ for $1 \leq j \leq m$,

$1 \leq i \leq \frac{n}{2}$ and $n$ is even,

$f\left(u_{2 i-1}^{j}\right)=1+2(s-1)(j-1)+4(i-1) \quad$ for $1 \leq j \leq t$,

$1 \leq i \leq \frac{s}{2}$ and $s$ is even,

$f\left(u_{2 i}^{j}\right)=4 m(n-1)+4 t(s-1)-1-2(s-1)(j-1)+4(i-1)$

for $1 \leq j \leq t, 1 \leq i \leq \frac{s}{2}$

and $s$ is even,

$f\left(v_{2 i-1}^{j}\right)=2+2 n(j-1)+4(i-1) \quad$ for $1 \leq j \leq m$,

$1 \leq i \leq \frac{n+1}{2}$ and $n$ is odd,

$f\left(v_{2 i}^{j}\right)=4 m n-4 m-2(n-2)(j-1)-4(i-1) \quad$ for $1 \leq j \leq m$,

$1 \leq i \leq \frac{n-1}{2}$ and $n$ is odd,

$f\left(u_{2 i-1}^{j}\right)=1+2 s(j-1)+4(i-1) \quad$ for $1 \leq j \leq t$,

$1 \leq i \leq \frac{s+1}{2}$ and $s$ is odd,

$f\left(u_{2 i}^{j}\right)=4 m(n-1)+4 t(s-1)-1-2(s-2)(j-1)+4(i-1)$

odd.

for $1 \leq j \leq t, 1 \leq i \leq \frac{s-1}{2}$ and $s$ is

For each vertex labeling, the induced edge label $f^{*}$ is defined as follows: $f^{*}\left(E\left(m P_{n} \cup t P_{s}\right)\right)=2 m(n-1)+2 t(s-1)-1-2(i-1): 1 \leq i \leq$ $m(n-1)+t(s-1)$.

It can be verified that $f$ is a skolem odd difference mean labeling of 
$m P_{n} \cup t P_{s}$.

Theorem 2.5. The graph $m K_{1, n} \cup t K_{1, s}$ is a skolem odd difference mean graph if $m, n, t, s \geq 1$.

Proof. Let $v_{1}^{1}, v_{2}^{1}, v_{3}^{1}, \ldots, v_{n}^{1}$ and $v_{1}^{2}, v_{2}^{2}, v_{3}^{2}, \ldots, v_{n}^{2}$ upto $v_{1}^{t}, v_{2}^{t}, v_{3}^{t}, \ldots, v_{n}^{t}$ and $u_{1}^{1}, u_{2}^{1}, u_{3}^{1}, \ldots, u_{s}^{1}$ and $u_{1}^{2}, u_{2}^{2}, u_{3}^{2}, \ldots, u_{s}^{2}$, upto $u_{1}^{m}, u_{2}^{m}, u_{3}^{m}, \ldots, u_{s}^{m}$ be the vertices of the graph $m K_{1, n} \cup t K_{1, s}$.

Let $E\left(m K_{1, n} \cup t K_{1, s}\right)=\left\{u_{1}^{j} u_{1+i}^{j}: 1 \leq i \leq n, 1 \leq j \leq m\right.$ and $\left.v_{1}^{j} v_{1+i}^{j}: 1 \leq i \leq s, 1 \leq j \leq t\right\}$.

Define $f: V\left(m K_{1, n} \cup t K_{1, s}\right) \rightarrow\{0,1,2, \ldots, 4(m n+t s)+m+t-3\}$ as follows:

$f\left(u_{1}^{j}\right)=2(j-1)$

$f\left(u_{1+i}^{j}\right)=4 m n+4 t s-2-4(i-1)-2(j-1)(2 n-1)$ and $1 \leq i \leq n$, $f\left(v_{1}^{j}\right)=(2 j-1)$ $f\left(v_{1+i}^{j}\right)=4 t s-1-4(i-1)-2(j-1)(2 s-1)$ and $1 \leq i \leq s$. for $1 \leq j \leq m$, for $1 \leq j \leq m$ for $1 \leq j \leq t$, for $1 \leq j \leq t$

For each vertex labeling, the induced edge label $f^{*}$ is defined as follows: $f^{*}\left(E\left(m K_{1, n} \cup t K_{1, s}\right)\right)=(2 i-1)$ for $1 \leq i \leq(m n+t s)$.

It can be verified that $f$ is a skolem odd difference mean labeling of $m K_{1, n} \cup t K_{1, s}$.

Theorem 2.6. If $G(p, q)$ is a skolem odd difference mean graph then $p \geq q$.

Proof. Suppose that $G$ is a skolem odd difference mean graph with $p$ vertices and $q$ edges and $p<q$. Let $f: V(G) \rightarrow\{0,1,2,3, \ldots, p+3 q-3\}$ be skolem odd difference mean labeling of $G$.

The induced edge labels are $\{1,3,5, \ldots, 2 q-1\}$.

Let $u v$ be an edge of $G$ with $f^{*}(u v)=2 q-1$. Take $f(u)=a$ and $f(v)=b$. Without loss of generality assume that $a>b$. Then $0 \leq a, b \leq p+3 q-3$. Now we consider the following two cases.

Case(i). $a-b$ is odd.

Then $\frac{|f(u)-f(v)|+1}{2}=\frac{a-b+1}{2}=2 q-1$. Hence, $a=4 q+b-3$. As, $a \leq p+3 q-3$ 
we have $q+b \leq p$. Since $p<q$, then $q+b<q$ which implies that $b<0$ which is a contradiction to $b \geq 0$.

Case(ii). $a-b$ is even.

Then $\frac{|f(u)-f(v)|}{2}=2 q-1$. Hence, $a=4 q-2+b \leq p+3 q-3$ which implies that $b+q \leq p-1$. Since $p<q$, then $b<-1$. This is a contradiction to $b \geq 0$.

Theorem 2.7. The wheel graph $W_{n}, n>2$ is not a skolem odd difference mean graph.

Proof. The wheel graph $W_{n}, n>2$ has $n+1$ vertices and $2 n$ edges. Suppose that $f$ is a skolem odd difference mean labeling of $W_{n}$. Hence the induced edge labels are $\{1,3, \ldots, 4 n-1\}$. Let $u v$ be an edge with $f^{*}(u v)=4 n-1$. Let $f(u)=a$ and $f(v)=b$. Without loss of generality assume that $a>b$. Then $0 \leq b<a \leq 7 n-2$.

If $\frac{|f(u)-f(v)|}{2}=4 n-1$ then $\frac{a-b}{2}=4 n-1$ which implies $a=b+8 n-2 \geq$ $8 n-2>7 n-2$.

If $\frac{|f(u)-f(v)+1|}{2}=4 n-1$ then $\frac{a-b+1}{2}=4 n-1$ which implies $a=b+8 n-3 \geq$ $8 n-3>8 n-2>7 n-2$.

This is a contradiction to $a, b \leq 7 n-2$. Hence the wheel graph $W_{n}, n>2$ is not a skolem odd difference mean graph.

Theorem 2.8. The umbrella graph $U_{n, m}, n>2$ and $m \geq 1$ is not a skolem odd difference mean graph.

Proof. The umbrella graph $U_{n, m}, n>2$ and $m \geq 1$, has $m+n$ vertices and $m+2(n-1)$ edges. Suppose that $f$ is a skolem odd difference mean labeling of $G$. Hence the induced edges are $\{1,3, \ldots, 2 m+4 n-5\}$.

Let $u v$ be an edge with $f^{*}(u v)=2 m+4 n-5$. Let $f(u)=a$ and $f(v)=b$. Without loss of generality assume that $a>b$. Then $0 \leq b<a \leq 4 m+7 n-9$. If $\frac{|f(u)-f(v)|}{2}=2 m+4 n-5$ then $\frac{a-b}{2}=2 m+4 n-5$ this implies that $a=b+4 m+8 n-10 \geq 4 m+8 n-10>4 m+8 n-9>4 m+7 n-9$.

If $\frac{|f(u)-f(v)+1|}{2}=2 m+4 n-5$ then $\frac{a-b+1}{2}=4 n-1$ this implies that $a=b+4 m+8 n-11 \geq 4 m+8 n-11>4 m+8 n-9>4 m+7 n-9$.

This is a contradiction to $a, b \leq 4 m+7 n-9$. Hence the umbrella graph $U_{n, m}, n>2$ and $m \geq 1$ is not a skolem odd difference mean graph.

Theorem 2.9. The graph $B_{n}$ is not a skolem odd difference mean graph if $n \geq 2$. 
Proof. If $n=1$ then $B_{1}$ is a cycle graph that admits skolem odd difference mean graph in [7]. If $n \geq 2$, the number of edges in $B_{n}$ graph is $q=3 n+1$. Therefore $p+3 q-3=11 n+2$.

To get $2 q-1=6 n+1$ as edge label, the minimum vertex label is $12 n+1$. But $11 n+2<12 n+1$ for all $n \geq 2$. Therefore $2 q-1$ cannot occur as an edge label of $B_{n}$ for $n \geq 2$. Hence $B_{n}$ is not a skolem odd difference mean graph if $n \geq 2$.

Theorem 2.10. The graph $L_{n}$ is not a skolem odd difference mean graph if $n \geq 3$.

Proof. If $n=2$ then $L_{2}$ is a cycle graph that admits skolem odd difference mean graph in [7]. The graph $L_{n}(n \geq 3)$ has $2 n$ vertices and $3 n-2$ edges.

For $n \geq 3, p+3 q-3=11 n-9$.

That is the maximum possible vertex label of $L_{n}$ is $11 n-9$.

Therefore, it is not possible to get an edge with label $2 q-1=6 n-5$.

Hence $L_{n}(n \geq 3)$ is not a skolem odd diference mean graph.

\section{References}

[1] F. Harary, Graph theory, Addison Wesley, Massachusetts, (1972).

[2] Joseph A. Gallian, A Dynamic Survey of Graph Labeling, The Electronic Journal of Combinatorics, (2015), \#DS6.

[3] K. Manickam and M. Marudai, Odd mean labelings of graphs, Bulletin of Pure and Applied Sciences, 25E (1), pp. 149-153, (2006).

[4] K. Murugan, A. Subramanaian, Skolem difference mean labeling of Hgraphs, International Journal of Mathematics and Soft Computing, 1, (1), pp. 115-129, (2011).

[5] D. Ramya and M. Selvi, On skolem difference mean labeling of some trees, International Journal of Mathematics and Soft Computing, 4 (2), pp. 11-18, (2014).

[6] D. Ramya, M. Selvi and R. Kalaiyarasi, On skolem difference mean labeling of graphs, International Journal of Mathematical Archive, 4 (12), pp. 73-79, (2013). 
[7] D. Ramya, R. Kalaiyarasi and P. Jeyanthi, On skolem odd difference mean labeling of graphs, Journal of Algorithms and Computing, (45), pp. 1-12, (2014).

[8] S. Somasundaram and R. Ponraj, Mean labelings of graphs, National Academy Science Letter, (26), pp. 210-213, (2003).

\section{P. Jeyanthi}

Research Centre, Department of Mathematics, Govindammal Aditanar College for Women, Tiruchendur-628215, Tamilnadu, India

e-mail : jeyajeyanthi@rediffmail.com

\section{R. Kalaiyarasi}

Department of Mathematics, Dr. Sivanthi Aditanar College of Engineering, Tiruchendur-628215, Tamilnadu, India e-mail : 2014prasanna@gmail.com

\section{Ramya}

Department of Mathematics, Government Arts College for Women, Ramanathapuram, Tamilnadu, India

e-mail : aymar_padma@yahoo.co.in

and

\section{T. Saratha Devi}

Department of Mathematics, G. Venkataswamy Naidu College, Kovilpatti-628502, Tamilnadu,

India

e-mail : rajanvino03@gmail.com 